\title{
NATIONAL PHYSICAL LABORATORY, TEDDINGTON
}

\author{
OPEN DAY
}

\section{$\mathrm{A}^{\mathrm{n}}$} FTER the extended series of Open Days held at the National Physical Laboratory last year in celebration of the Laboratory's jubilee, the corresponding function this year was confined to a single Clay, May 23, which was also made the occasion of the annual visitation and meeting of the General Board. In a day of ideal summer weather, nearly one thousand visitors were entertained at the Laboratory. The number of items of research on show was much less than was exhibited last year; but more than one-quarter was concerned with new work.

In the Light Division, a demonstration was given of a development which has recently created considerable interest, namely, transparent electrically conducting films on glass. As a result of methods developed at the Laboratory, it is possible to deposit on glass exceedingly fine films of gold, of the order of a quarter of a millionth of an inch thick, which have an electrical resistance as low as $10 \mathrm{ohms}$ between the opposite sides of a square sample, and an optical transmission as high as about 80 per cent. These results are closely reproducible, so that, although these effects have so far been produced only on small areas of glass, the extension of the idea for the purpose of de-icing aircraft windows and like applications appears to be possible. Also in this Division, further exhibits were shown of recent developments in the manufacture of diffraction gratings by the Merton-N.P.L. process. Gratings so far produced with five thousand lines to the inch are finding a ready application in the field of infrared spectroscopy, where they are replacing costly prisms of such materials as lithium and calcium fluoride in the work of exploring molecular structure.

Several demonstrations were given by the Aerodynamies Division which are of particular importance in the subject of high-speed flight. One of the problems being investigated concerns the effect which may be produced when the boundary layer on a wing or other body moving at high speeds meets a shock wave. In these circumstances it may thicken or separate from the surface, and thus the shape and strength of the shock wave may be modified. Interaction problems of this type are important, and it is essential to understand them in order to allow for the scale effects which result from the use of smallscale models in high-speed wind tunnels. Detailed studies of this type of interaction have been in progress at the Laboratory for several years, and recently a small wind tunnel has been built in which the range of Mach and Reynolds number which can be covered is greater than had been possible previously. The Mach number can be varied from $1 \frac{1}{2}$ to 4 by changing the shape of the nozzle used to produce the supersonic flow. The stagnation pressure of the air entering the tunnel can be varied between $\frac{1}{2}$ and 20 atmospheres absolute, thus giving Reynolds numbers from $0.5 \times 10^{8}$ to $20 \times 10^{6}$ on the flat plate. Results obtained so far indicate that changes of the Mach or Reynolds number have a considerable effect on the interaction. Another section of the work concerns research on delta and arrowhead forms of fighter aircraft. An arrowhead wing was shown in the low- turbulence tunnel, where it was forced to oscillate in pitch by applying a reciprocating force through a spring; movements in the wings were recorded photographically. 'This work is of importance as this particular type of aircraft, especially when travelling at high speeds, is liable to give rise to undamped pitching oscillations, which make the machine unstable and difficult to control.

The Electricity Division had on show a method for the precise measurement of dielectric constants of pure liquids. These measurements are being carried out in the first instance on benzene, which is being regarded as a standard liquid. The measuring cell consists essentially of electrodes in the form of concentric tubes of platinum fitted with a guard ring, which are mounted in a glass container. Benzene, which is supplied in a highly purified form, is distilled direct into the cell after it has undergone treatment to remove traces of dissolved gases and water. The capacitance of the cell is measured when the dielectric medium is first air (or a vacuum) and then benzene, and the ratio of one capacitance to the other gives the dielectric constant of the benzene. The measurements are being made in the first place at $1,000 \mathrm{c} . / \mathrm{s}$. and over a temperature range of $5-30^{\circ} \mathrm{C}$. The High Voltage Laboratory has continued work on the measurement of surge voltages, and a divider for use in such measurements was shown. These dividers can be constructed from resistors or capacitors or combinations of both; but, whichever is used, the essential requirement is that the wave-shape of rapidly changing phenomena should be faithfully produced at the oscillograph with a reduction ratio which can be accurately determined. The chief source of error for all forms of divider arises from the earth capacitance of the high-voltage arm; but the effects of inductive residuals in the low- and highvoltage arms cannot be neglected for surges occupying a microsecond or so.

The pilot model of the Automatic Computing Engine which was designed and made in the Electronics Section of the National Physical Laboratory has now been transferred to the Mathematics Division, where it is being used for high-speed computational work. Although the pilot model was originally intended merely as the forerunner of the final machine, it has proved to be particularly useful and will continue to be used while an engineered version and also the final machine are being produced.

In the Metallurgy Division a new method was demonstrated for the preparation of small specimens for tensile tests. In the preparation of specimens of the order of $1 / 16 \mathrm{in.}$ in diameter, the strain produced in the surface layer by machining is important, although for larger specimens it can usually be ignored. The problem of obtaining small specimens by some other method was solved by making the specimen the anode in a rather unusual electrolytic cell. A jet of suitable solution plays on the blank while it is rapidly rotated and at the same time moved to and fro along its axis at a uniform speed. A current passed between the blank as the anode and a cathode in the jet causes the blank to be 
dissolved away gradually and uniformly until the desired size is reached. If the current density is high enough and the electrolyte suitable, a truly electropolished surface is produced which is free from irregularities under a high-power microscope, as well as being free from strain. Another exhibit shown by the Metallurgy Division concerned the production of titanium. Although titanium is the fourth most abundant metal in the earth's crust and is widely distributed, it is only since the development of the magnesium-reduction process within the past decade that the metal has shown industrial potentialities. Previously it had not been possible to prepare the metal in a sufficiently pure state, and its true properties are shown only when in this condition. Research is being carried out at the Laboratory on new methods of preparation of the metal and on the constitution of titanium-oxygen and titanium-ironoxygen alloys. In connexion with research on atomic structure, an X-ray spectrometer capable of dealing with large and small specimens has recently been constructed in the Metallurgy Division. In this instrument, the X-ray intensity is recorded by a Geiger-Müller counter which can be positioned to an angular accuracy of \pm 17 seconds of arc. The pulses in the counter are amplified and recorded, and in this way quantitative measurements of diffraction maxima can be achieved in a fraction of the time required to expose and process a photographic plate. To eliminate errors due to variation of the X-ray source, a monitor Geiger-Müller tube is used for measuring the beam incident on the specimen. When the monitor tube has received a predetermined number of counts, it automatically stops the diffraction tube, the count of which thus corresponds to a fixed quantity of $\mathrm{X}$-radiation on the specimen. The overall accuracy attainable is about 0.5 per cent.

Demonstrations were given in the Metrology Division of emitters of highly reproducible waves of light which are being used in connexion with the measurement of distances of up to $0.5 \mathrm{~m}$. to an accuracy corresponding to one-millionth of an inch in ten inches. These sources use pure isotopes either of mercury or of krypton, both of which are now obtainable in sufficient quantity. During recent months, four N.P.L. gauges, the largest being $10 \mathrm{~cm}$. long, have been sent to two other standards laboratories in Europe for measurement in terms of these wave-lengths of light. The results obtained at the three laboratories are in very good agreement, all the measured values for each gauge lying within a range of $\pm 1 \times 10^{-6} \mathrm{in}$. Measurements are also continuing with radio waves $12.5 \mathrm{~mm}$. in length for the purpose of being able to measure up to distances of about $\mathbf{1 . 6} \mathrm{m}$. by interferometry. The accuracy so far attained is of the order of $0.0001 \mathrm{in}$., and the technique which has been developed shows considerable promise of extension to distances of $30 \mathrm{~m}$. or more. A re-determination of the density of mercury is to be carried out in connexion with the National Physical Laboratory standard barometer, and in order to do this a $3 \frac{1}{2}$-in. cube of sintered tungsten carbide has been prepared in the Metrology Division workshop with a standard of workmanship which is thought to be unsurpassed. The faces of the cube have been lapped to within $10 \times 10^{-6} \mathrm{in}$. of flatness, and a little short of this in parallelism, while the edges and corners have also been carefully finished. It is hoped that this perfection of geometric form will permit the volume to be determined from interferometric measurements of the linear dimensions. 'The cube will then be weighed in air and in mercury in which, by virtue of its density, it will just sink. The accuracy which it is hoped to attain corresponds to better than $0.001 \mathrm{~mm}$. of mercury in the measurement of atmospheric pressure and less than $0.0001^{\circ} \mathrm{C}$. in the measurement of temperature at the steam point.

In the Physics Division a new method has been devised for measuring the elastic properties of materials over a temperature-range from near the absolute zero to above red heat. In the new equipment the specimen is lightly suspended in the centre of a long tube and either cooled by surrounding it by cold liquids jacketed in a special form of Dewar flask, or else heated by electrical means. The specimen has attached to it long nickel filaments which can be used for setting it into oscillation and also for measuring the oscillations, since nickel has the property of changing magnetic variations into vibrations and vice versa. The precision obtainable is of the order of 0.03 per cent in favourable circumstances, and thus enables a knowledge of the elastic moduli and of the anelastic behaviour to be obtained. This information is of particular value because of the present tendency for materials, such as turbine blades, to be stressed very highly, even at temperatures in excess of $1,000^{\circ} \mathrm{C}$. In another part of the Physics Division, a new Smith bridge has been built for the purpose of measuring normal temperatures to an accuracy of $\pm 0.0001^{\circ} \mathrm{C}$.

In the Ship Division, developments have taken place in the technique for cutting propellers. In the latest method thin steel templates are first cut from an enlarged drawing through a pantograph mechanism, a scale ratio of $5: 1$ being employed. Then the templates are clamped around cylindrical formers in such a way that they give a correct skeleton replica of the face or back of the screw. The formers are mounted on a shaft carrying the serew, and this shaft is rotated and advances so that a follower maintains contact with the template while an electrically driven bandcutter traces the correct section on a metal screw blank. On the final version of the machine it is hoped to cut all four blades at once. A new torsionmeter which had been designed and made in the former Engineering Division workshop was also shown. Although the operating principle is similar to that of the commercial type of instrument, the deflexion is transmitted from one end of the gauge length to the other by means of a small tube lying parallel to the shaft instead of by a shell concentric with it. Thus a very considerable reduction in the size and weight of the instrument has been achieved. The instrument is carried on hardened points bearing on the shaft and is clamped to it by chains; a range of shaft diameters of 14-22 in. can be accommodated. Experiments are being carried out in the water tunnel on the effect on the cavitation characteristics of a ship's screw arising from a non-uniform velocity distribution of the water. Gauzes of varying mesh inserted in the tunnel circuit are used for producing the appropriate variation in velocity.

In the High Temperature Mechanical Properties Section, an investigation is in progress on the variation of fatigue strength over a speed-range of 10-8,000 cycles per minute on a mild steel for temperatures up to $500^{\circ} \mathrm{C}$. It has been found particularly important to investigate the effects resulting from the use of the higher frequencies.

Visitors were also able to inspect the various items of routine test work in the Test House and to discuss problems connected with the maintenance of standards of quality. 